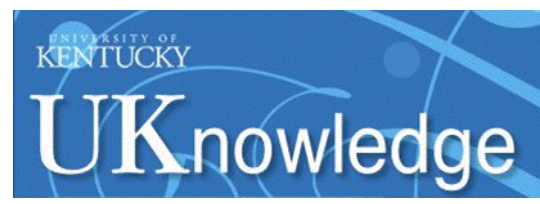

University of Kentucky

UKnowledge

\title{
Dust and PAH Emission in the Star-Forming Active Nucleus of NGC 1097
}

\author{
R. E. Mason \\ Northern Operations Center
}

N. A. Levenson

University of Kentucky

C. Packham

University of Florida

Moshe Elitzur

University of Kentucky, moshe@pa.uky.edu

J. Radomski
Gemini Observatory, Chile

See next page for additional authors

Follow this and additional works at: https://uknowledge.uky.edu/physastron_facpub

Part of the Astrophysics and Astronomy Commons, and the Physics Commons

Right click to open a feedback form in a new tab to let us know how this document benefits you.

\section{Repository Citation}

Mason, R. E.; Levenson, N. A.; Packham, C.; Elitzur, Moshe; Radomski, J.; Petric, A. O.; and Wright, G. S., "Dust and PAH Emission in the Star-Forming Active Nucleus of NGC 1097" (2007). Physics and Astronomy Faculty Publications. 206.

https://uknowledge.uky.edu/physastron_facpub/206

This Article is brought to you for free and open access by the Physics and Astronomy at UKnowledge. It has been accepted for inclusion in Physics and Astronomy Faculty Publications by an authorized administrator of UKnowledge. For more information, please contact UKnowledge@lsv.uky.edu. 


\section{Dust and PAH Emission in the Star-Forming Active Nucleus of NGC 1097}

Digital Object Identifier (DOI)

http://dx.doi.org/10.1086/512356

\section{Notes/Citation Information}

Published in The Astrophysical Journal, v. 659, no. 1, p. 241-249.

(c) 2007. The American Astronomical Society. All rights reserved.

The copyright holder has granted permission for posting the article here.

Authors

R. E. Mason, N. A. Levenson, C. Packham, Moshe Elitzur, J. Radomski, A. O. Petric, and G. S. Wright 


\title{
DUST AND PAH EMISSION IN THE STAR-FORMING ACTIVE NUCLEUS OF NGC 1097
}

\author{
R. E. MASON \\ Gemini Observatory, Northern Operations Center, Hilo, HI 96720; rmason@gemini.edu \\ N. A. LeVenson \\ Department of Physics and Astronomy, University of Kentucky, Lexington, KY 40506; levenson@pa.uky.edu \\ C. PACKHAM \\ Department of Astronomy, University of Florida, Gainesville, Fl 32611; packham@astro.ufl.edu \\ M. Elitzur \\ Department of Physics and Astronomy, University of Kentucky, Lexington, KY 40506; moshe@pa.uky.edu \\ J. RADOMSKI \\ Gemini Observatory, Southern Operations Center, La Serena, Chile; jradomski@gemini.edu
}

A. O. Petric

Astrophysics Department, Columbia University, New York, NY 10025; andreea@astro.columbia.edu

AND

G. S. WRIGHT

UK Astronomy Technology Center, Royal Observatory Edinburgh, Edinburgh, EH9 3HJ, UK; gsw@roe.ac.uk Received 2006 August 5; accepted 2007 January 4

\begin{abstract}
The nucleus of the nearby galaxy NGC 1097 is known to host a young, compact $(r<9 \mathrm{pc})$ nuclear star cluster, as well as a low-luminosity active galactic nucleus (AGN). It has been suggested both that the nuclear stellar cluster is associated with a dusty torus and that low-luminosity AGNs like NGC 1097 do not have the torus predicted by the unified model of AGNs. To investigate these contradictory possibilities we have acquired Gemini/T-ReCS 11.7 and $18.3 \mu$ m images of the central few hundred parsecs of this galaxy at $<45 \mathrm{pc}$ angular resolution, in which the nucleus and spectacular, kiloparsec-scale star-forming ring are detected in both bands. The small-scale mid-IR luminosity implies thermal emission from warm dust close to the central engine. Fitting of torus models shows that the observed mid-IR emission cannot be accounted for by dust heated by the central engine. Rather, the principal source heating the dust in this object is the nuclear star cluster itself, suggesting that the detected dust is not the torus of AGN unified schemes (although it is also possible that the dusty starburst itself could provide the obscuration invoked by the unified model). Comparison of Spitzer IRS and Gemini GNIRS spectra shows that, although PAH bands are strong in the immediate circumnuclear region of the galaxy, PAH emission is weak or absent in the central $19 \mathrm{pc}$. The lack of PAH emission can probably be explained largely by destruction/ionization of PAH molecules by hard photons from the nuclear star cluster. If NGC 1097 is typical, PAH emission bands may not be a useful tool with which to find very compact nuclear starbursts even in low-luminosity AGNs.
\end{abstract}

Subject headings: dust, extinction — galaxies: active — galaxies: individual (NGC 1097) — galaxies: nuclei — infrared: galaxies

\section{INTRODUCTION}

The dusty molecular torus, cornerstone of the unified model of active galactic nuclei (AGNs), has been the subject of much observational and theoretical scrutiny in recent years. The detection of broad emission lines in polarized light in a number of type 2 objects (Antonucci \& Miller 1985; Miller \& Goodrich 1990; Young et al. 1996; Moran et al. 2000; Tran 2001) demonstrates the overall validity of the unified model, and structures thought to correspond to the torus itself have now been resolved through mid-IR interferometry of one Seyfert galaxy (Jaffe et al. 2004). However, questions remain as to the origin and precise nature of the obscuration and the extent to which it can account for the differences between the many classes of AGNs.

In particular, the possibility that the torus and star formation are intimately connected has been raised by several authors on theoretical grounds. For instance, the material in the nucleus needs to have a certain scale height to obscure the nucleus and account for the relative numbers of type 1 and 2 AGNs, and it has been suggested that the energy necessary to maintain this thickness might be supplied by supernovae in nuclear starbursts (Wada \& Norman 2002). Indeed, some or all of the nuclear obscuration has been proposed to arise in dusty clouds ejected from such starbursts (Cid Fernandes \& Terlevich 1995; Fabian et al. 1998; Watabe \& Umemura 2005) or in optically thick stellar winds (Zier \& Biermann 2002). Observationally, young stars are found quite commonly in the inner few hundred parsecs of AGNs (e.g., González Delgado et al. 1998; Storchi-Bergmann et al. 2000; Imanishi 2002; Imanishi 2003; Rodríguez-Ardila \& Viegas 2003).

However, while few-hundred-parsec-scale star formation is often observed in AGNs, few young stellar clusters have to date been detected at smaller scales. The outer diameter of the torus remains rather unclear, but its warm, mid-IR-emitting regions have not yet been resolved in single-dish 10-20 $\mu$ m observations of even the nearest objects (e.g., Bock et al. 2000; Radomski et al. 2003; Soifer et al. 2003; Packham et al. 2005; Mason et al. 2006), 
and interferometric measurements of NGC 1068 suggest that its mid-IR-emitting regions are only $\sim 3$ pc $\left(\sim 0.04^{\prime \prime}\right)$ in diameter (Jaffe et al. 2004). Near-IR adaptive optics imaging of the Circinus galaxy (Prieto et al. 2004) resolves a source of FWHM $\sim 2 \mathrm{pc}$, consistent with a parsec-scale torus at those wavelengths.

The discovery by Storchi-Bergmann et al. (2005, hereafter SB05) of UV absorption signatures of a $10^{6} M_{\odot}, 10^{6}$ year old stellar cluster within only $9 \mathrm{pc}$ of the nucleus of the nearby LINER/ Seyfert 1 galaxy NGC $1097^{1}$ is therefore of obvious interest. The star cluster-but not the broad emission lines from the nucleus itself - suffers about 3 mag of visual extinction, consistent with the possibility of starburst activity physically associated with a dusty AGN torus. Young superstellar clusters or recent star formation are also known to exist in the central few parsecs of a handful of other AGNs (Colina et al. 2002; Davies et al. 2006b; Mueller Sánchez et al. 2006).

While the results of SB05 are suggestive of a starburst genuinely associated with an obscuring torus, the observation that many LINERs host nuclear UV point sources raises some doubt as to whether LINER-type nuclei in fact host obscuring tori (Maoz et al. 2005). This is expected on theoretical grounds in the context of the disk-wind scenario for the torus: below a certain luminosity the accretion onto the black hole can no longer sustain the mass outflow necessary to obscure the nucleus (Elitzur \& Shlosman 2006). Moreover, how the properties of LINER tori, if they exist, might relate to those observed in Seyfert galaxies is completely open to question. Further observations of LINER nuclei to probe their detailed nuclear structure are therefore desirable.

In addition, the UV evidence of star formation detected by SB05 is very susceptible to extinction, so UV detections of starbursts in AGN tori are likely to be limited to cases of nuclei in which the stellar clusters are not too deeply buried within the nuclear dust. Interpreting the energetics of UV-detected starbursts will be complicated by uncertain extinction corrections. A more useful tracer of the nuclear starburst phenomenon would be an infrared star formation indicator that is both unaffected by dust extinction and accessible from the ground, to take advantage of the spatial resolution achievable from ground-based telescopes. The $3.3 \mu \mathrm{m}$ polycyclic aromatic hydrocarbon (PAH) emission band fits both of these criteria. Usually attributed to vibrational relaxation of aromatic hydrocarbon molecules excited by far-UV photons, the PAH bands have been used extensively as tracers of star formation in extragalactic environments (e.g., Moorwood 1986; Roche et al. 1991; Genzel et al. 1998; Lutz et al. 1998; Rigopoulou et al. 1999; Laurent et al. 2000; Tran 2001; Imanishi \& Wada 2004; Peeters et al. 2004; Risaliti et al. 2006).

The suite of PAH emission features is commonly detected in large-aperture Infrared Space Observatory (ISO) spectra of nearby active galaxies with circumnuclear star-forming regions (Rigopoulou et al. 1999), but on moving to apertures of $3^{\prime \prime}-5^{\prime \prime}$ ( $\sim$ few hundred parsecs) or less, the bands are often weak or absent (Roche et al. 1991, 2006, 2007; Le Floc'h et al. 2001; Siebenmorgen et al. 2004). Modeling suggests that the carriers of the bands are easily destroyed by the harsh radiation field close to the active nucleus itself (Roche \& Aitken 1985; Siebenmorgen et al. 2004; Voit 1992). However, this appears not always to be the case. For instance, Imanishi \& Wada (2004) find that the surface brightness of the nuclear PAH emission in Seyfert 1 galaxies is comparable to that in starburst galaxies, and the $3.3 \mu \mathrm{m}$

\footnotetext{
${ }^{1}$ Although NGC 1097 hosts a classic LINER nucleus (Phillips et al. 1984), broad, double-peaked emission lines in its spectrum are characteristic of rotating gas in an AGN accretion disk (Storchi-Bergmann et al. 1993).
}

PAH band has been shown to be present in several Seyfert galaxies of both types 1 and 2 on fairly small spatial scales, including, in the case of NGC 3227, within only $60 \mathrm{pc}$ of the central engine (Rodríguez-Ardila \& Viegas 2003). To exist at such small distances from these AGNs, it seems likely that the fragile PAH band carriers are shielded from the nuclear X-rays. This intriguing result could perhaps be explained if the carriers exist within the dense, dusty clouds of the torus itself.

To further investigate this possibility, we have performed L-band ( 3-4 $\mu \mathrm{m})$ spectroscopy of the nucleus of NGC 1097 $\left(D=17 \mathrm{Mpc}\right.$ for $\left.H_{0}=75 \mathrm{~km} \mathrm{~s}^{-1} \mathrm{Mpc}^{-1} ; 1^{\prime \prime}=85 \mathrm{pc}\right)$ to search for the $3.3 \mu \mathrm{m}$ PAH emission band. We have also obtained images at 11.7 and $18.5 \mu \mathrm{m}$, to search for the compact mid-IR source expected to be a signature of the torus and to constrain its properties. The data were taken using the Thermal Region Camera and Spectrograph (T-ReCS) and the Gemini Near-Infrared Spectrograph (GNIRS) on the Gemini South $8.1 \mathrm{~m}$ telescope, yielding $\leq 0.52^{\prime \prime}$ spatial resolution and therefore probing distances $\leq 22 \mathrm{pc}$ from the nucleus. We also discuss complementary Spitzer Space Telescope Infrared Spectrograph (IRS) observations of the central $3.6^{\prime \prime} \times 3.6^{\prime \prime}$ (approximately $300 \times 300 \mathrm{pc}$ ) of this galaxy. Section 2 describes all of these observations and the reduction of the data. In $\S 3$ we present the nuclear data and describe the characteristics of the compact source detected in the mid-IR, and compare our subarcsecond-resolution data and that of Prieto et al. (2005) with the clumpy torus models of Nenkova et al. (2002). The conclusions that can be drawn from this work are discussed in $\S 4$.

\section{OBSERVATIONS AND DATA REDUCTION}

\subsection{Mid-IR Imaging}

Narrowband N $(11.7 \mu \mathrm{m})$ and Q $(18.3 \mu \mathrm{m})$ band images of NGC 1097 were taken on 2005 September 16 and 17 using the Gemini South mid-IR instrument T-ReCS (Telesco et al. 1998). T-ReCS uses a Raytheon $320 \times 240$ pixel $\mathrm{Si}: \mathrm{As}$ IBC array, providing a plate scale of $0.089^{\prime \prime}$ per pixel. The detector was used in correlated quadruple sampling mode (Sako et al. 2003). Images were obtained in the $11.7 \mu \mathrm{m}(\delta \lambda=1.13 \mu \mathrm{m}, 50 \%$ cut-on/ cutoff $)$ and $18.3 \mu \mathrm{m}(\delta \lambda=1.51 \mu \mathrm{m}, 50 \%$ cut-on/cutoff $)$ filters using the standard chop-nod technique to remove time-variable sky background, telescope thermal emission, and " $1 / f$ " noise. The chop throw was $15^{\prime \prime}$, and the telescope was nodded $15^{\prime \prime}$ in the direction of the chop every $30 \mathrm{~s}$. The chop angle was chosen based on the $5^{\prime \prime}$ aperture N-band data of Telesco \& Gatley (1981) to minimize the extended emission in the off-source beam and was fixed at $90^{\circ}$ east of north. The total on-source time for the 11.7 and $18.3 \mu \mathrm{m}$ observations was 456 and $912 \mathrm{~s}$, respectively

The data were reduced using the Gemini IRAF package. The difference for each chopped pair for each nod set was calculated, and the nod sets were then differenced and combined until a single image was created. Chopped pairs obviously compromised by variable sky background, high electronic noise, or other problems were searched for, but no data needed to be discarded.

The Cohen standards SAO 216405 and SAO 193679, as well as PPM 278407, were observed for flux and point-spread function (PSF) calibration. Observations of the standards taken approximately $1 \mathrm{hr}$ apart showed a variation in counts of around $10 \%$, typical of mid-IR photometry. The PSF observations were made immediately before or after the NGC 1097 observations and with the same instrumental configuration. The FWHM of the PSF star was $0.41^{\prime \prime}$ (standard deviation $\sim 0.03^{\prime \prime}$ ) at $11.7 \mu \mathrm{m}$ and $0.52^{\prime \prime}$ in the $18.3 \mu \mathrm{m}$ filter. Short PSF or flux standard observations are comparable to longer source observations as the closed-loop active optics of Gemini provides a similar PSF when taken at a similar 
telescope pointing and time (see e.g., Radomski et al. 2003; Packham et al. 2005 although seeing variations may of course affect the final observed PSF). As judged by the FWHM of the standard stars, the PSF was stable over the course of these observations.

\subsection{L-Band Spectroscopy}

L-band spectroscopy of the nucleus of NGC 1097 was obtained using GNIRS, the near-IR spectrograph on the Gemini South Telescope, on 2005 November 13. The short red camera and 3 pixel $\left(0.45^{\prime \prime}\right)$ slit were used together with the $321 \mathrm{~mm}^{-1}$ grating, resulting in $R \sim 1100$. The total integration time was 30 minutes, and the telescope was nodded by $20^{\prime \prime}$ every $30 \mathrm{~s}$ in an ABBA pattern so that the sky background emission could be subtracted out. Sky conditions were photometric during these observations.

The nucleus was acquired in the $H$ band to take advantage of the contrast between object and sky at those wavelengths. A point source contributes $52 \%$ of the $H$-band flux in a $0.4^{\prime \prime}$ diameter aperture, that fraction increases with wavelength (Prieto et al. 2005), and we observe an unresolved source at $11.7 \mu \mathrm{m}$ (§3.1), suggesting that the location at which the slit was centered at $1.6 \mu \mathrm{m}$ also corresponds to the flux peaks at 3.3 and $11.7 \mu \mathrm{m}$. The slit was orientated at $9^{\circ}$ east of north in order to encompass one of the circumnuclear emission knots visible in the T-ReCS image, but that knot proved too faint to yield a useful spectrum and will not be considered further.

The data were reduced using the Gemini IRAF package in combination with standard IRAF and Starlink FIGARO routines. After bad pixel masking, flat-fielding, and subtracting in pairs, the individual sky-subtracted spectra were averaged together, with the positive and negative beams cross-correlated and shifted in both the spatial and dispersion directions before combining. The central three pixels $\left(0.45^{\prime \prime}\right)$ of the galaxy spectrum were extracted using the standard star spectrum to define the curvature of the extraction aperture across the array.

Wavelength calibration was accomplished using the numerous telluric absorption lines in the spectrum. The galaxy spectrum was then cross-correlated with the spectrum of the standard star, BS 466 (F7 V), and divided by that spectrum. In order to improve the cancellation of telluric lines, the resulting spectrum was multiplied by a logarithmically scaled model atmospheric spectrum, ${ }^{2}$ binned to the appropriate spectral resolution. This had the effect of slightly reducing the amplitude of some of the residuals in the $3.3 \mu \mathrm{m}$ region. The spectrum was then multiplied by a $6240 \mathrm{~K}$ blackbody curve. Flux calibration was with reference to BS 466, using a $V$-band magnitude of 6.24 and $V-\mathrm{L}=1.36$. No attempt was made to correct for uncertain differential slit losses between the galaxy and standard star (due to e.g., seeing variations and different intrinsic spatial flux distributions) and, as is usual for flux calibration of narrow-slit spectral data, the uncertainty of the derived flux density is considerable.

\subsection{IRS Spectroscopy}

Publicly available spectra of the nucleus of NGC 1097 taken with the Short-Low (first-order) module of the Spitzer Space Telescope's Infrared Spectrograph (Houck et al. 2004 IRS) were retrieved from the Spitzer data archive (PID 14; PI Houck). The data have been published in a study of the global properties of

\footnotetext{
${ }^{2}$ Lord (1992) obtained from http://www.gemini.edu/sciops/ObsProcess/ obsConstraints/ocTransSpectra.html.
}

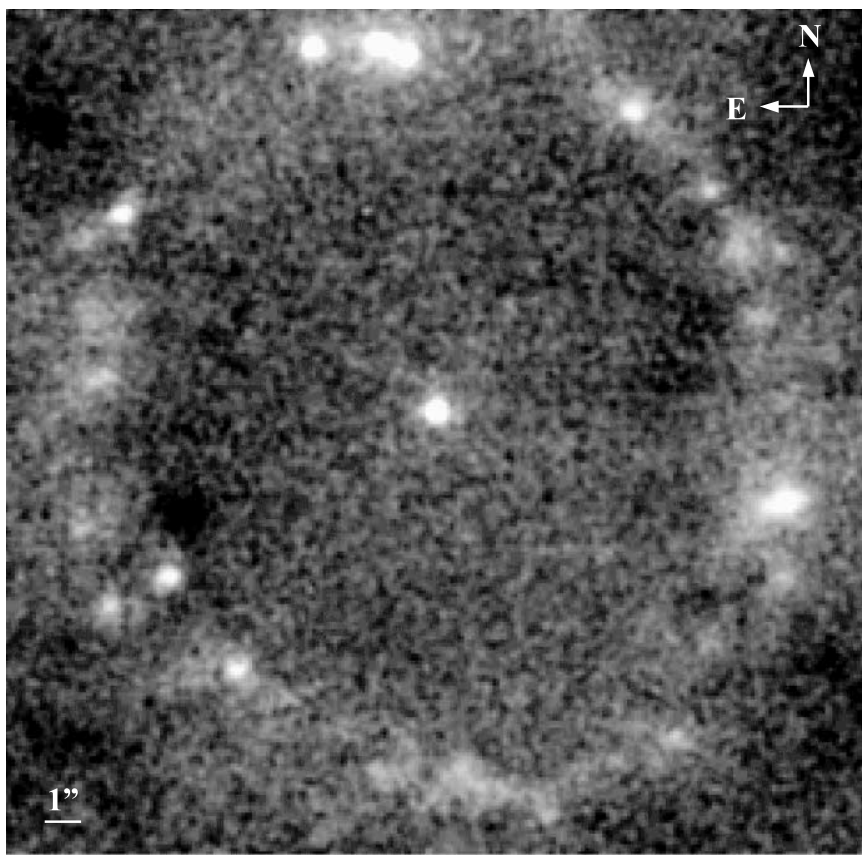

FIG. 1.-Central portion of the T-ReCS 11.7 image of NGC 1097, smoothed with a 2 pixel Gaussian. The (unguided) negative off-source images are evident at either side of the positive galaxy image.

starburst galaxies by Brandl et al. (2006). These $R \sim 100$ observations represent a total integration time of $56 \mathrm{~s}$. Files output from the IRS pipeline at the Spitzer Science Center (which includes ramp fitting, dark-sky subtraction, droop correction, linearity correction, flat-fielding, and wavelength calibration) had background light subtracted by subtracting the first-order slit exposed during second-order observations of NGC 1097 taken a few minutes previously. Spectra were extracted from the background-subtracted, co-added frames using SPICE, the Spitzer Custom Extractor. The extraction window scales with wavelength but was defined to be 2 pixels $\left(3.6^{\prime \prime}\right)$ at $11.2 \mu \mathrm{m}$, equal to the $3.6^{\prime \prime}$ slit width and comparable to Spitzer's diffraction limit at $11.2 \mu \mathrm{m}\left(3.3^{\prime \prime}\right)$.

The spatial profile of the IRS spectrum shows flux between the nucleus and the circumnuclear ring $\sim 10^{\prime \prime}$ distant, presumably from the wings of the PSFs of these bright sources combined with real diffuse emission from that region. Neither the point source nor the extended source flux calibration provided by SPICE is optimized to deal with this situation. However, the conclusions drawn from these observations do not depend critically on a highly accurate calibration, so we simply normalize the mean flux in the IRS spectrum to that given by Telesco \& Gatley (1981) over a similar bandpass in a slightly larger aperture (5" diameter vs. $\left.3.6^{\prime \prime} \times 3.6^{\prime \prime}\right)$.

\section{RESULTS}

\subsection{The Mid-IR Images and Model Fits}

The T-ReCS mid-IR images of NGC 1097 are shown in Figures 1 and 2. A compact nuclear source and the well-known circumnuclear star-forming ring are clearly detected at both 11.7 and $18.3 \mu \mathrm{m}$. The starburst ring emits strongly in the mid-IR, with each of the three knots in the northern part of the ring contributing a similar level of flux to that of the nucleus itself. The $15^{\prime \prime}$ maximum chop throw of the Gemini telescopes means that the positive and negative images partially overlap in the region of the circumnuclear ring. Inside the ring, faint extended emission is very probably present but is difficult to detect without a clear 


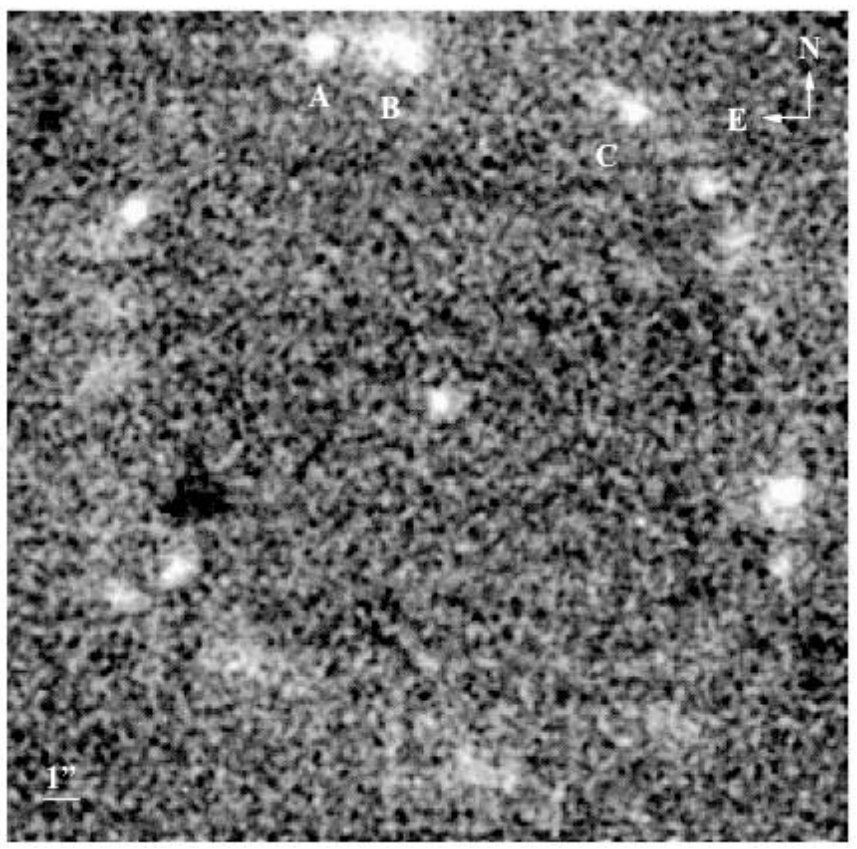

FIG. 2.- Same as for Fig. 1, but at $18.3 \mu \mathrm{m}$. Photometry of clumps A, B, and $\mathrm{C}$ is given in Table 1 .

sample of the sky on which to measure the zero level of the background. The flux of the nuclear point source is approximately $48 \mathrm{mJy}$ at $11.7 \mu \mathrm{m}$ and $64 \mathrm{mJy}$ at $18.3 \mu \mathrm{m}$ (Table 1), roughly $75 \%$ and $27 \%$ of the broadband, $5^{\prime \prime}$ aperture measurements of Telesco \& Gatley (1981) at N and Q, respectively, and consistent with the $60 \mathrm{mJy}, 1.5^{\prime \prime}$ aperture N-band upper limit of Gorjian et al. (2004). Faint emission between the nucleus and ring likely accounts for the difference between our data and that of Telesco \& Gatley (1981) most clearly affecting the Q-band photometry.

Table 1 gives the flux densities of the three regions of emission in the circumnuclear ring that are clearly detected at $18.3 \mu \mathrm{m}$ and least likely to be affected by the negative chop beams. The aperture sizes used were determined partly by the proximity of neighboring emission regions and/or the edge of the detector. The N/Q flux density ratios are in the region of 0.4 for the ring, but rather higher, about 0.75 , for the nucleus.

The FWHM of the central compact source was measured to be $0.43^{\prime \prime}$ at $11.7 \mu \mathrm{m}$, comparable to the mean FWHM of the comparison star observations $\left(0.41^{\prime \prime} \pm 0.03^{\prime \prime} ; \S 2\right)$. This translates to a diameter of $<37 \mathrm{pc}$, consistent with the near-IR limit of $10 \mathrm{pc}$ diameter of Prieto et al. (2005). Thus, like all AGNs observed noninterferometrically to date, the nuclear source of NGC 1097 is not resolved at its FWHM in the N band. The limited signal-tonoise ratio prevented a meaningful measurement of the source size at $18.3 \mu \mathrm{m}$.

While AGN- or star-formation-heated dust is expected to emit in the mid-IR, other processes can also contribute to the nuclear mid-IR emission. For instance, as shown by Perlman et al. (2001) and Whysong \& Antonucci (2004), the $10 \mu \mathrm{m}$ emission from M87 on subarcsecond scales can be fully accounted for by synchrotron radiation from the inner regions of the radio jet. However, the thin disk + jet + radiatively inefficient accretion flow (RIAF) model of Nemmen et al. (2006), which does not include any thermal dust emission, predicts mid-IR luminosity around an order of magnitude lower in both the $\mathrm{N}$ and $\mathrm{Q}$ bands than that observed from the unresolved nuclear source in NGC 1097 , $\log \left[\nu L_{\nu}\left(\mathrm{erg} \mathrm{s}^{-1}\right)\right] \approx 41.6$ at both $\mathrm{N}$ and $\mathrm{Q}$ for $D=17 \mathrm{Mpc}$. Altering the mass accretion rate or other parameters of the thin disk + RIAF model cannot account for the observed mid-IR flux, given the observational constraints at shorter wavelengths. Even allowing for the fact that the jet parameters in the Nemmen et al. model are not well constrained, we conclude that the bulk of the mid-IR emission that we detect comes from warm dust in the region of the central engine.

To investigate the contribution of the AGNs to the heating of the nuclear dust, we fit the observed fluxes with the inhomogeneous torus models of Nenkova et al. (2002). In these computations, individual clouds are optically thick, and the inner radius of their toroidal distribution is located at the dust sublimation radius. While the total extent of the torus may reach 30-100 times the inner radius, the smallest physical scales, where the clouds are hottest, dominate the emission at near/mid-IR wavelengths. The physical parameters that describe the distribution of clumps include the average number of clouds along radial rays in the equatorial plane, $N_{0}$, and the optical depth per cloud in the $V$ band, $\tau_{V}$. The clouds follow a power-law distribution in radius, $r$, with number declining as $r^{-q}$. The mid-IR emission is not sensitive to the outer radius of the cloud distribution, so we fix it at 30 times the inner radius.

We require that the clump distribution give a high probability $(>0.5)$ of direct views of the AGNs, given the lack of reddening of the broad $\mathrm{H} \alpha$ line and the low X-ray column density toward the nucleus (Terashima et al. 2002; Nemmen et al. 2006). Storchi-Bergmann et al. (2003) find the inclination angle of the

TABLE 1

Photometry of the Nucleus and Circumnuclear Ring of NGC 1097

\begin{tabular}{|c|c|c|c|}
\hline Location $^{\mathrm{a}}$ & $\begin{array}{l}\text { Aperture Diameter } \\
(\operatorname{arcsec})\end{array}$ & $\begin{array}{c}11.7 \mu \mathrm{m} \text { Flux Density } \\
(\mathrm{mJy})\end{array}$ & $\begin{array}{c}18.3 \mu \mathrm{m} \text { Flux Density } \\
(\mathrm{mJy})\end{array}$ \\
\hline Nucleus ................. & $3.0^{\mathrm{b}}$ & 48 & 64 \\
\hline А & 1.6 & 34 & 83 \\
\hline В & 2.0 & 75 & 169 \\
\hline $\mathrm{C}$ & 1.6 & 25 & 63 \\
\hline
\end{tabular}

a See Fig. 2.

b An aperture of $3^{\prime \prime}$ diameter was used for the photometry of the nucleus, chosen based on the profiles of the standard stars to ensure that all of the flux from the unresolved source was collected. A surrounding "sky" annulus was used to subtract off any residual sky background and diffuse emission surrounding the nucleus. The (unknown) spatial variation of surrounding diffuse emission will therefore determine the degree to which the photometry represents the unresolved source alone, but as any such emission must be close to the sensitivity limit of the instrument, we expect it to make a negligible contribution to the measured nuclear flux. 


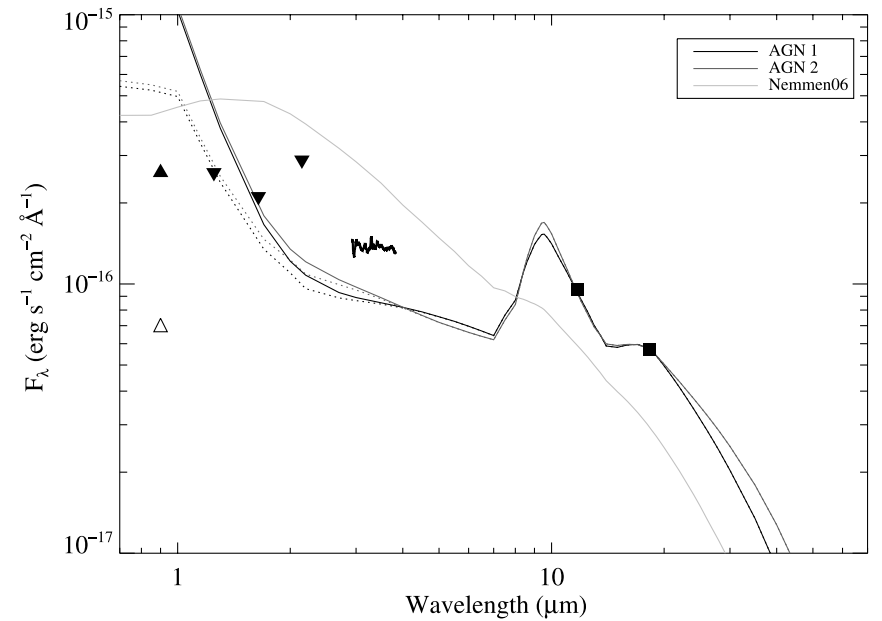

FIG. 3.- Inhomogeneous dusty torus models, fit only to the 11.7 and $18.3 \mu \mathrm{m}$ fluxes (squares) and constrained to allow a direct view of the central engine. The sum of the intrinsic heating spectrum and the resulting dust emission is plotted as a solid line in each case. Several different obscuring geometries could produce the mid-IR emission if a standard AGN spectrum (black and dark gray lines) heats the dusty clouds. These AGN models are scaled to the $18.3 \mu \mathrm{m}$ flux, and applying foreground extinction of the intrinsic source of $A_{V}=0.12 \mathrm{mag}$ results in the corresponding dotted lines. Alternatively, using the fit of Nemmen et al. (2006) as the heating spectrum, none of the allowed torus geometries gives a good fit to the mid-IR flux ratio or the observed flux in either band. We plot the best of these (light gray line), fixed to the intrinsic luminosity determined by Nemmen et al. (2006). None of these AGN or RIAF solutions remains consistent with the observations at all wavelengths. We plot a smoothed version of the L-band spectrum (thick line) and small-scale near-IR "AGN magnitudes" (inverted triangles; see text). We also show the total observed emission ( filled triangle) and reddened starburst contribution (open triangle) that SB05 measure at $9000 \AA$ A. ISO and Infrared Astronomical Satellite (IRAS) data, which could in principle constrain the models in the far-IR, are not shown as the large apertures involved contain a very large contribution from emission from the bright, kiloparsec-scale star-forming ring. See the text for a complete description of the different models.

accretion disk $i=34^{\circ}{ }^{3}$ We assume that the torus is aligned with the accretion disk and adopt $i=30^{\circ}$ in the coarse model grid. The torus does not have a sharp edge, but rather a Gaussian angular distribution away from the equatorial plane of width $\sigma$. In terms of the angle from the equatorial plane, $\beta$, the number of clouds along a ray $N(\beta)=N_{0} \exp \left(-\beta^{2} / \sigma^{2}\right)$. To allow direct views to the nucleus with the viewing angle constrained, we consider $\sigma=30^{\circ}$, which roughly corresponds to a torus half-opening angle of $60^{\circ}$, and $\sigma=45^{\circ}$ (for half-opening angle $\sim 45^{\circ}$ ). With the broader distribution, the probability of viewing the unobscured nucleus is generally low, and only $N_{0} \leq 2$ is acceptable at $i=30^{\circ}$.

We consider three different heating spectra and fit the sum of the intrinsic spectrum and the resulting dusty emission to the ratio of our N-and Q-band fluxes. We scale the results to the flux at Q (Figs. 3 and 4), except when using the result of Nemmen et al. (2006) for the input spectrum, in which case multiwavelength observations already constrain the luminosity. Also shown in Figures 3 and 4 are "AGN magnitudes," the $J-, H$-, and $K$-band flux densities of the nuclear point source derived by fitting unresolved emission and an underlying galaxy component to near-IR imaging data (Prieto et al. 2005). ${ }^{4}$ We speculate that NGC 1097 will re-

\footnotetext{
3 Note that contrary to the description in their paper, Storchi-Bergmann et al. (2003) intend $i$ to have its usual meaning, measured with respect to the rotation axis (not the plane) of the disk, so edge-on views have $i=90^{\circ}$ (M. Eracleous 2006, private communication).

4 The values given in Prieto et al. (2005) are erroneous; corrected values were supplied by M. A. Prieto (2006, private communication).
}

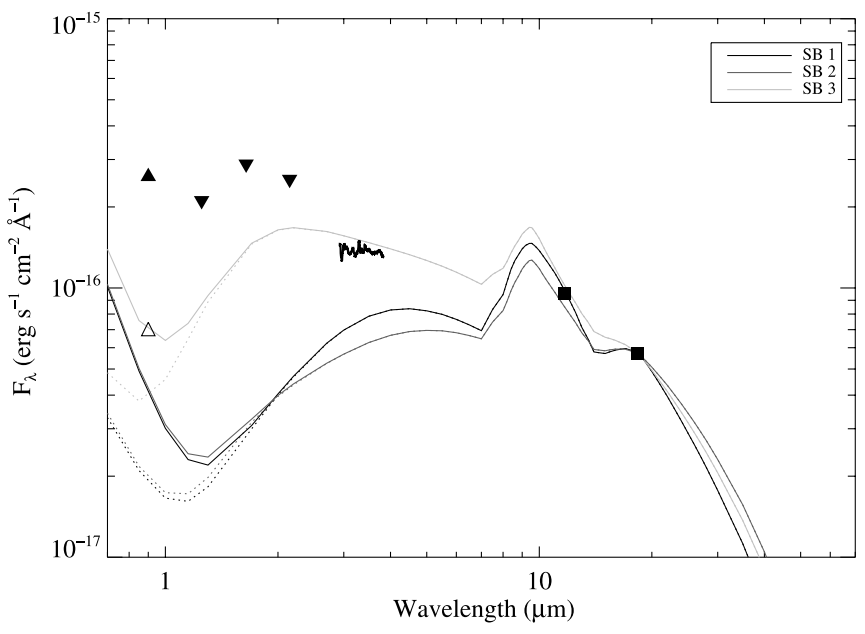

FIG. 4.-Fits of the starburst-heated dust model to the SED of NGC 1097. Solid lines show the sum of the intrinsic spectrum and the dust-reprocessed emission. Dotted lines show extinction of the starburst spectrum by $A_{V}=3$. The starburst emission peaks in the UV, so it heats the dust effectively while not contributing directly to the net IR flux. Data as for Fig. 3, and model details are listed in the text.

main unresolved in the mid-IR at resolutions similar to the PSF used by Prieto et al. (2005) to fit the near-IR data. Such a result would imply that the mid-IR emitting region is $\lesssim 10 \mathrm{pc}$ in radius, as has been found for other nearby AGNs (e.g., Soifer et al. 2003; Jaffe et al. 2004; Packham et al. 2005; Roche et al. 2006). We therefore believe it likely that these near-IR data sample the same spatial region as our mid-IR points.

The first dust-heating spectrum we consider is that of a standard AGN (in which $\lambda F_{\lambda} \propto \lambda^{-0.5}$ from 0.1 to $1 \mu \mathrm{m}$ ). Although the low observed optical and UV fluxes together with the minimal obscuration along the line of sight rule out this model for NGC 1097, it serves as a useful point of comparison. In this case, the dusty material dominates the mid-IR emission, even when the AGN is viewed directly, and the AGN itself dominates at optical and near-IR wavelengths. The dust sublimation radius scales with the square root of the bolometric luminosity of the heating source and is a function of the heating spectrum. This AGN heating spectrum and the observed $L_{\mathrm{bol}}=$ $10^{42} \mathrm{ergs} \mathrm{s}^{-1}$ set the inner radius at $0.01 \mathrm{pc}$. The observed N/Q ratio and a likely unimpeded view of the central engine exclude $q=1,2$, and 3 radial distributions. Two reasonable models both have $q=0, \sigma=30^{\circ}$, and $N_{0}=2$, with $\tau_{V}=40$ and 100 in models "AGN 1" and "AGN 2," respectively. Scaling to the observed flux at Q yields $L_{\mathrm{bol}}=4 \times 10^{42} \mathrm{ergs} \mathrm{s}^{-1}$, which is a few times higher than the observed AGN luminosity. More severely, they significantly exceed the observed optical emission, even after correcting for the measured extinction. This implies that although dust heating by a standard AGN radiation field can reproduce the mid-IR spectral energy distribution (SED) of NGC 1097 well, this cannot in fact be the dominant dust-heating mechanism in this galaxy.

Nemmen et al. (2006) present the broadband SED of the nuclear region of NGC 1097. The data reveal the intrinsic spectrum at optical and UV wavelengths, which is responsible for heating the surrounding dust, although they do not constrain it well in the IR. Motivated by the empirical result at short wavelengths, we consider Nemmen et al.'s thin disk + jet + RIAF model fit to the data as an alternate heating source of the nuclear dust. We use this spectral model (which we refer to as the "Nemmen06" model for simplicity) as the input to the clumpy torus distribution of clouds. 
The Nemmen06 spectrum is weaker than the standard AGN at wavelengths below $1 \mu \mathrm{m}$ and relatively stronger than the standard AGN at longer wavelengths. The UV-weak continuum heats the clouds inefficiently, so the dust sublimation temperature is reached closer to the central source than in the standard AGN model of the same luminosity. With the Nemmen06 heating and $L_{\mathrm{bol}}=$ $10^{42} \mathrm{ergs} \mathrm{s}^{-1}$, the inner radius is located at $0.003 \mathrm{pc}$, while still scaling with the square root of luminosity. Fixing the viewing angle $\left(i=30^{\circ}\right)$, none of the $\sigma=30^{\circ}$ models fits the data. Several of the $\sigma=45^{\circ}$ models with $q=0$ or 1 agree with the mid-IR flux ratio. However, the required direct view to the AGN rules out these models, which have $N_{0}=20$, so the probability of an unobscured view of the nucleus is only 0.03 . For comparison with the standard AGN results, we show the best of the allowed models based on the Nemmen06 spectrum, in which $q=0$, $\sigma=45^{\circ}, N_{0}=2$, and $\tau_{V}=60$. We adopt the observed luminosity of the input Nemmen06 spectrum and therefore do not scale the result to the mid-IR data as we did with the standard AGN model. The resulting model, including the dust emission, underestimates the flux density at Q by a factor of 2 , while overestimating the flux density at $\mathrm{L}$ by a factor of 2 .

Both the standard AGN and Nemmen06 spectra produce similar SEDs of the torus alone, with a peak in $F_{\lambda}$ around $20-40 \mu \mathrm{m}$. However, because the total model is the sum of the input and torus spectra, the significant differences between the input spectra strongly affect the relative mid-IR contribution of the torus. The intrinsic AGN spectrum is very weak in the mid-IR, so the total mid-IR emission of this model is essentially that of the torus alone. Here the torus is thousands of times brighter than the input spectrum in the mid-IR, even when the torus scale height is small. In contrast, the Nemmen06 continuum is strong at nearand mid-IR wavelengths, and its torus does not provide more than 3 times the flux input at 11.7 or $18.3 \mu \mathrm{m}$. Consequently, these models generally overpredict the observed N/Q flux ratio. In the Nemmen06 case, the entire resulting optical to mid-IR spectrum is very sensitive to the input spectrum. The original model was not constrained well in the IR, and even without dust reprocessing it overpredicts the observed near-IR nuclear emission. A more severe problem of the Nemmen06-heated models is that the optical/mid-IR flux ratio is always too high. The optical/ mid-IR signature of the intrinsic spectrum persists here, even when the dust-reprocessed contribution is included. Fundamentally, because the short-wavelength flux is relatively weak, it cannot produce the strong mid-IR emission we measure. Thus, we conclude that the complete Nemmen06 model does not describe the IR data well. Changing the input contribution of the RIAF spectrum alone and neglecting dust-reprocessed emission is not a viable solution. The family of RIAF models cannot produce the observed mid-IR flux density, even when the underlying physical parameters of the models are varied.

That the clumpy torus models with neither a standard AGN spectrum nor the Nemmen06 spectrum are able to simultaneously reproduce the mid-IR and the near-IR/optical emission of NGC 1097 suggests that the nuclear starburst contributes to the intrinsic luminosity that the dusty clouds reprocess. In fact, a $10^{6} M_{\odot}$, $10^{6}$ year old starburst scaled by 0.85 (SB05) has $L_{\text {bol }}=5 \times$ $10^{42} \mathrm{ergs} \mathrm{s}^{-1}$ (Leitherer et al. 1999), several times higher than the AGN. To test this possibility we have adapted the clumpy torus models to use the young starburst spectrum for their input source, as an approximation to the mixed regions of dust and young stars presumably present in the nucleus.

We use a spherically symmetric distribution of clouds with $N_{0}$ fixed at 0.7 , for $P>0.5$ of a direct view of the central

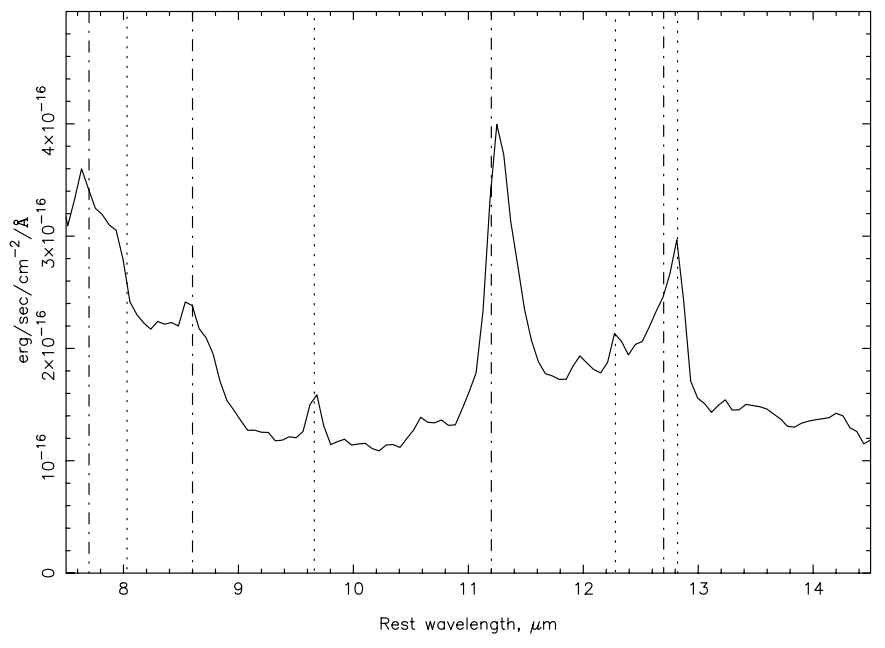

FIG. 5.-Spitzer IRS spectrum of the nucleus NGC 1097. Lines are drawn at $7.7 \mu \mathrm{m}$ (PAH), $8.03 \mu \mathrm{m}$ [ $\left.\mathrm{H}_{2} 0-0(\mathrm{~S} 4)\right], 8.6 \mu \mathrm{m}$ (PAH), $9.66 \mu \mathrm{m}\left[\mathrm{H}_{2} 0-0(\mathrm{~S} 3)\right]$, $11.2 \mu \mathrm{m}$ (PAH), $12.28 \mu \mathrm{m}$ [ $\mathrm{H}_{2} 0-0$ (S2)], $12.7 \mu \mathrm{m}(\mathrm{PAH})$, and $12.82 \mu \mathrm{m}$ ([ $\mathrm{Ne}$ II] $)$. The peak wavelengths of the PAH bands vary somewhat from source to source (van Diedenhoven et al. 2004).

engine. The observed N/Q ratio rules out $q=2$ and 3 solutions, but we find good solutions with $q=0-1$ and $\tau_{V}=20-60: q=0$ and $\tau_{V}=20$ ("SB 1" model), $q=0$ and $\tau_{V}=40$ ("SB 2" model), and $q=1$ and $\tau_{V}=60$ ("SB 3" model). These three models have $L=1.2,1.2$, and $1.6 \times 10^{42} \mathrm{ergs} \mathrm{s}^{-1}$, respectively, when the model output is scaled to the observed mid-IR flux. This is somewhat lower than the starburst luminosity of SB05, but we note that that value relies heavily on the large extinction correction of the UV spectrum. Because we fit only the mid-IR data, changing the extinction would not alter our preferred model fits, although it would change the modeled observable optical emission (Fig. 4, dotted lines). The starburst heats the dust effectively because of its high UV luminosity, but its weakness in the infrared relative to both the standard AGN power law and RIAF spectra means that the near-IR emission is predominantly from the dusty clouds, rather than from a bright AGN component that overpredicts the emission at optical wavelengths. The geometry of the starburst models is somewhat artificial, and we have no direct measurement of the spatial relationship between the starburstheated clouds and the accretion disk. All the flux measurements include both the starburst and the central engine along with the emission they reprocess on scales of $10 \mathrm{pc}$. Nevertheless, basic considerations of energetics and the broadband spectra of heating sources, as well as the specific model fits, suggest that the nuclear starburst is the dominant contributor to the heating of the dust in the central few tens of parsecs of the galaxy.

More detailed modeling would show more precisely the roles of the starburst and AGN in the heating of the dust. For example, we have opted against including any contribution heated by the central engine while fitting the starburst to avoid the near-IR problems inherent in the underlying RIAF spectrum. In this simplified model fitting, we have used only the N/Q ratio to determine the model parameters. A further complication that is beyond the scope of this work would be to fit a broader spectral range and to include luminosity constraints simultaneously. Nonetheless, even the N/Q ratio by itself robustly excludes large portions of parameter space. We emphasize that whatever its ultimate power source, the mid-IR emission alone demonstrates that warm dust is present in the nucleus of NGC 1097. The dust is local to the nucleus and must obscure it from certain lines of sight while leaving 


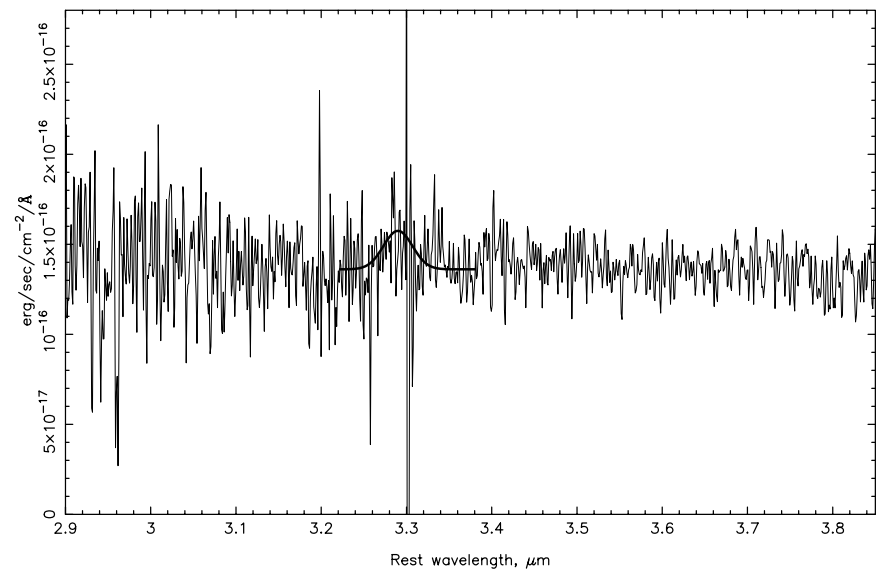

FIG. 6.-L-band spectrum of NGC 1097. The black line represents the strongest $3.3 \mu \mathrm{m}$ PAH band likely to be present in the spectrum (see text).

the broad lines unobscured from our viewing angle, fulfilling at least two of the requirements of the AGN unified scheme.

\subsection{The Spectra}

The IRS short-low spectrum of NGC 1097 (Fig. 5) shows a number of prominent emission features, including the 7.7, 8.6, 11.2, and $12.7 \mu \mathrm{m}$ PAH bands (this last line blended with the $12.82 \mu \mathrm{m}[\mathrm{Ne}$ II] fine-structure line). NGC 1097 is therefore one of the growing number of AGNs in which strong PAH emission is detected within as little as $150 \mathrm{pc}$ of the active nucleus.

To estimate the $3.3 \mu \mathrm{m}$ band flux that would be expected from the $3.6^{\prime \prime} \times 3.6^{\prime \prime}$ (approximately $300 \times 300 \mathrm{pc}$ ) region covered by the IRS spectrum, we use the finding of Hony et al. (2001) that the $11.2 \mu \mathrm{m}$ band is typically a factor of $3-4$ stronger than the $3.3 \mu \mathrm{m}$ feature in a wide range of environments. The flux in the $11.2 \mu \mathrm{m}$ band, $7.1 \times 10^{-13}$ ergs s${ }^{-1} \mathrm{~cm}^{-2}$ (as obtained by a spline fit to the continuum and direct integration under the band), then implies $\sim 2 \times 10^{-13} \mathrm{ergs} \mathrm{s}^{-1} \mathrm{~cm}^{-2}$ in the $3.3 \mu \mathrm{m}$ feature. The corresponding luminosities are $2.5 \times 10^{40}$ and $\sim 7 \times 10^{39} \mathrm{ergs} \mathrm{s}^{-1}$ for the 11.2 and $3.3 \mu \mathrm{m}$ bands, respectively, within about a factor of 2 of the $3.3 \mu \mathrm{m}$ PAH luminosity of Seyfert 1 galaxies such as NGC 4235 and MCG -2-33-34 on similar physical scales (Imanishi \& Wada 2004).

In the GNIRS spectrum of the central $0.45^{\prime \prime} \times 0.45^{\prime \prime}$, however, no $3.3 \mu \mathrm{m}$ feature is evident (Fig. 6). Using the $95 \%$ confidence interval on the difference between the means of points near the peak of and just outside the feature (and assuming a Gaussian profile with FWHM $=0.04 \mu \mathrm{m}$; van Diedenhoven et al. 2004), we estimate the $3.3 \mu \mathrm{m}$ PAH flux to be $\lesssim 9 \times 10^{-15} \mathrm{ergs} \mathrm{s}^{-1} \mathrm{~cm}^{-2}$. This is well below the flux that would be expected if the carriers of the $11.2 \mu \mathrm{m}$ band were concentrated in the unresolved mid-IR source, as might be expected if they existed predominantly in warm, dense, dusty nuclear clouds, even allowing for uncertainties in the flux calibration of the GNIRS and IRS spectra. The spatial distribution of the mid-IR and PAH emission is very different.

Comparison of the N-band and $\mathrm{L}^{\prime}$-band continuum fluxes in similar apertures ( Telesco \& Gatley 1981; Alonso-Herrero et al. 2001), combined with the above $11.2 / 3.3 \mu \mathrm{m}$ band ratio, suggests that $3.3 \mu \mathrm{m}$ band emission would peak at nearly twice the continuum level in our spectrum if the PAH band- and continuumemitting material were similarly distributed over the central region of NGC 1097. That would imply an equivalent width $\left(\mathrm{EW}_{3.3}\right) \sim$ $40 \mathrm{~nm}$, whereas the maximum likely $3.3 \mu \mathrm{m}$ feature flux estimated above implies $\mathrm{EW}_{3.3} \lesssim 7 \mathrm{~nm}$. This puts the $\mathrm{EW}_{3.3}$ of the nuclear starburst in NGC 1097 at the low end of the values reported for few-hundred-parsec-scale starbursts by Imanishi (2003), although comparable to several of those in Imanishi \& Wada (2004). The lack of PAH emission in the nucleus implies an increase in the relative level of featureless L-band continuum in this aperture, possibly due to destruction of the PAH molecules (and/or dehydrogenation and ionization, which affect the molecules' ability to emit at $3.3 \mu \mathrm{m}$ and their likelihood of photodissociation; Allain et al. 1996; Allamandola et al. 1999). This is examined in more detail in $\S 4.2$.

\section{DISCUSSION}

\subsection{The Nuclear Mid-IR Emission}

The detection of UV variability, hard X-ray spectra and polarized broad emission lines in a number of LINERs (e.g., Barth et al. 1999; Terashima \& Wilson 2003; Maoz et al. 2005) has demonstrated that at least some of these objects harbor accreting supermassive black holes at their centers. An obvious question, then, is whether these LINERs possess the dusty tori postulated by the AGN unified scheme.

In the case of NGC 1097, the detection of unresolved thermal emission implies that there is warm dust in the nucleus of this galaxy that would obscure the nuclear broad lines if viewed from certain angles. As with Seyfert nuclei, the dust is compact $(r<19 \mathrm{pc})$ at mid-IR wavelengths. On these two counts at least, it satisfies the requirements of the AGN unified model. On the other hand, our modeling shows that the accretion-powered nucleus of NGC 1097 is incapable of heating the dust efficiently enough to produce the observed mid-IR emission. The torus demanded by the unified model, if it exists at all, should be about a factor of 2 weaker in the mid-IR than the entity that we have detected, and have a different SED. The dust that we observe is not heated by the central engine and is therefore not necessarily directly associated with the AGN, consistent with the prediction of Elitzur \& Shlosman (2006) that tori should disappear at $L<10^{42}$ ergs s$^{-1}$.

This does not entirely rule out, however, that NGC 1097 hosts a dusty torus. If a cool, IR-faint torus previously existed, Figure 4 shows that the young nuclear star cluster is capable of heating the dust so that it emits quite strongly in the mid-IR. Alternatively, the starburst itself could be the obscurer of the unified model. For this to be plausible, the dust associated with the starburst must provide only partial covering and star formation must be common in the innermost regions of at least low-luminosity AGNs. The geometry of the dust is not well constrained by the observations and modeling presented here, but Prieto et al. (2005) detect cold, dusty filaments spiraling down into the central $10 \mathrm{pc}$ of NGC 1097, apparently in a thin-disk geometry, and a kinematic study by Fathi et al. (2006) shows material streaming into this central $10 \mathrm{pc}$ region. Fathi et al. (2006) suggest that the infalling material may have triggered the nuclear star formation, and disks of fairly recently formed stars seem quite common in the central $\sim 50$ pc of Seyfert galaxies (Davies et al. 2006a). Obscuration by dusty starbursts seems at odds with the fact that low-luminosity AGNs appear to have unobscured nuclei (about as many of the 17 LINERs with known nuclear UV point sources are type $2 \mathrm{~s}$ as type 1s; Maoz et al. 2005), but could still apply to some fraction of the population. An observational assessment of the frequency of very small-scale star formation in these objects would be valuable in this context.

To the best of our knowledge, small-scale nuclear dust emission has been searched for in only a handful of other LINERs (Chary et al. 2000; Grossan et al. 2001; Perlman et al. 2001; 
Whysong \& Antonucci 2004), of which such emission was detected only in M81. Grossan et al. (2001) note that the SED of M81 resembles those of Seyfert galaxies and quasars, suggesting that the nuclear $10 \mu \mathrm{m}$ emission in that object is also from a warm, central dust concentration. These results show that at least some LINERs do have dusty nuclei, but it is not yet clear whether these galaxies, particularly NGC 1097 with its nuclear starburst and infalling material, are unusual cases. High spatial resolution infrared observations of a larger sample of objects would help to establish whether or not this is true of AGN-containing LINERs in general, elucidate the mechanisms governing the presence or absence of the dust, and clarify how these low-luminosity objects may fit into unified schemes of AGNs.

\subsection{The Lack of Strong PAH Emission at the Nucleus}

As discussed in $\S 3.2,3.3 \mu \mathrm{m}$ PAH emission is not detected in the nucleus of NGC 1097, although a strong $11.2 \mu \mathrm{m}$ band is seen in the larger aperture IRS spectrum (Figs. 5 and 6). Most of the L-band flux in the smaller aperture must arise in material that does not produce a $3.3 \mu \mathrm{m}$ band and whose emission becomes more important in smaller apertures. Likely sources of such emission include hot dust around the AGN and nuclear stellar cluster, and the central engine itself. The intrinsic AGN/ RIAF contribution to the L-band spectrum is not taken into account in Figure 4, but it would have to account for most of the total in order to "dilute" the PAH emission enough to avoid its detection in the GNIRS spectrum. The model fits suggest that this is unlikely (most of the L-band emission is thermal), even taking into account the simplicity of the starburst model, but some contribution is certainly possible. As for hot dust, that dust must be depleted (relative to the surrounding regions) in PAH molecules able to emit at $3.3 \mu \mathrm{m}$.

Both the AGN and starburst may contribute to suppressing the PAH emission. Calculations suggest that PAH molecules should be destroyed close to luminous AGNs; in particular, Siebenmorgen et al. (2004) estimate that the PAH band carriers should be destroyed by soft X-ray fluxes $\gtrsim 10^{3} \mathrm{ergs} \mathrm{s}^{-1} \mathrm{~cm}^{-2}$. Extending the spectral model of Nemmen et al. (2006) and correcting for extinction, the intrinsic AGN luminosity from 0.02 to $2 \mathrm{keV}$ is $6.2 \times 10^{40} \mathrm{ergs} \mathrm{s}^{-1}$. At a distance of $150 \mathrm{pc}$ from the nucleus - the most distant regions covered by the IRS spectrumthe flux is then only about $10^{-2} \mathrm{ergs} \mathrm{s}^{-1} \mathrm{~cm}^{-2}$, consistent with the appearance of the PAH bands in the Spitzer data. Even at only $1 \mathrm{pc}$, however, the (current) soft X-ray flux from the AGN is a factor of a few below the PAH destruction threshold.

While the starburst does not provide much X-ray emission, it is luminous at UV wavelengths. The $10^{6} M_{\odot}, 10^{6}$ year old starburst has an intrinsic luminosity above $0.02 \mathrm{keV}$ of $2.1 \times 10^{42} \mathrm{ergs} \mathrm{s}^{-1}$ (Leitherer et al. 1999). Because of the starburst's softer radiation field, the average flux must exceed $\sim 10^{4} \mathrm{ergs} \mathrm{s}^{-1} \mathrm{~cm}^{-2}$ to destroy PAHs (Siebenmorgen et al. 2004). The starburst would be unlikely to destroy the distant molecules detected in the IRS spectrum, but it could destroy the PAHs located within $\sim 1 \mathrm{pc}$.

Observations of other stellar clusters of similar mass and age support the idea that the stellar cluster itself is partly responsible for the lack of PAH emission in its immediate surroundings. Tacconi-Garman et al. (2005) recently showed that, while the peak $3.3 \mu \mathrm{m}$ band flux occurs in the general nuclear regions of the starburst galaxies NGC 253 and NGC 1808, the feature-tocontinuum ratio actually reaches a minimum at the locations of maximum star formation activity in those nuclei. This is ascribed to photoionization or photodestruction of the PAH molecules in the radiation field of the stellar clusters. The strong PAH emis- sion from the massive, young clusters in galaxies like He 2-10 (Alonso-Herrero et al. 2004) might simply arise in diffuse material outside the immediate vicinity of the star clusters but still included in the $1.6^{\prime \prime}$ slit used for that work; a similar explanation has been suggested for the PAH bands in the Antennae galaxies (Snijders et al. 2006). This would account for the strength of the PAH bands in the Spitzer spectrum of NGC 1097 and their weakness at the nucleus.

To summarize, the $3.3 \mu \mathrm{m}$ band has been a useful tracer of star formation on fairly small scales ( $\sim 60$ to a few hundred parsecs) in AGNs (Imanishi 2002, 2003; Rodríguez-Ardila \& Viegas 2003; Imanishi \& Wada 2004). However, if NGC 1097 turns out to be typical, the combined effects of AGN continuum and the radiation field of stellar clusters themselves probably mean that it is unlikely that the $3.3 \mu \mathrm{m}$ feature will be as useful a diagnostic of vigorous star formation occurring on the smallest (few parsec) scales.

\section{CONCLUSIONS}

We have performed mid-IR imaging and L-band spectroscopy of the central region of NGC 1097, at $\lesssim 0.5^{\prime \prime}$ angular resolution, to search for thermal dust emission from the active nucleus and to test a potential diagnostic of small-scale nuclear star formation. A compact nuclear source is detected at both 11.7 and $18.3 \mu \mathrm{m}$, with a luminosity well in excess of that predicted by the accretion disk + RIAF + jet model of Nemmen et al. (2006). This mid-IR "excess" is strong evidence for thermal emission from dust in the nucleus of this low-luminosity AGN.

Fitting clumpy obscuration models to this and other highresolution IR data suggests that the star cluster detected by StorchiBergmann et al. (2005), not the central engine, is the dominant contributor to the heating of the dust in the nucleus. In fact, tori heated solely by low-luminosity AGNs - if they exist at allare likely to be relatively faint even in the mid-IR close to where their thermal emission peaks. This may mean that the mid-IR emission that we detect is not from the torus of AGN unified schemes, in accordance with certain predictions that tori should not exist in AGNs with luminosities as low as that of NGC 1097. Alternatively, it is possible, although not required by the data, that dust associated with the small-scale nuclear starburst is itself the obscuring material of the unified model. Further observations of IR emission and signatures of nuclear star formation in low-luminosity AGNs would help distinguish between these possibilities.

Despite the likely energetic importance of the starburst, the $3.3 \mu \mathrm{m}$ PAH band is not detected in the central $19 \mathrm{pc}$ of this galaxy, although strong $11.2 \mu \mathrm{m}$ PAH emission is observed in the surrounding areas. Destruction and/or ionization of the PAH molecules in the radiation field of the stellar cluster itself are likely to account for the nondetection of the $3.3 \mu \mathrm{m}$ band. These factors may limit the use of PAH features as extinction-insensitive tracers of very compact nuclear starbursts associated with dusty tori in other low-luminosity AGNs.

We would like to thank A. Alonso-Herrero, M. Eracleous, T. Geballe, and M. A. Prieto for providing helpful information, and the anonymous referee for a careful reading of the manuscript. N. A. L. acknowledges work supported by the National Science Foundation under grant 0237291. C. P. acknowledges work supported by the National Science Foundation under grant 0206617. This paper is based on observations obtained at the Gemini Observatory (Program ID: GS-2005B-Q-29), which is operated by 
the Association of Universities for Research in Astronomy, Inc., under a cooperative agreement with the NSF on behalf of the Gemini partnership: the National Science Foundation (United States), the Particle Physics and Astronomy Research Council (United Kingdom), the National Research Council (Canada),
CONICYT (Chile), the Australian Research Council (Australia), CNPq (Brazil), and CONICET (Argentina). This work is also based in part on observations made with the Spitzer Space Telescope, which is operated by the Jet Propulsion Laboratory, California Institute of Technology under a contract with NASA.
Allain, T., Leach, S., \& Sedlmayr, E. 1996, A\&A, 305, 616

Allamandola, L. J., Hudgins, D. M., \& Sandford, S. A. 1999, ApJ, 511, L115

Alonso-Herrero, A., Engelbracht, C. W., Rieke, M. J., Rieke, G. H., \& Quillen, A. C. 2001, ApJ, 546, 952

Alonso-Herrero, A., Takagi, T., Baker, A. J., Rieke, G. H., Rieke, M. J., Imanishi, M., \& Scoville, N. Z. 2004, ApJ, 612, 222

Antonucci, R. R. J., \& Miller, J. S. 1985, ApJ, 297, 621

Barth, A. J., Filippenko, A. V., \& Moran, E. C. 1999, ApJ, 525, 673

Brandl, B. R., et al. 2006, ApJ, 653, 1129

Bock, J. J., et al. 2000, AJ, 120, 2904

Chary, R., Becklin, E. E., Evans, A. S., Neugebauer, G., Scoville, N. Z., Matthews, K., \& Ressler, M. E. 2000, ApJ, 531, 756

Cid Fernandes, R. J., \& Terlevich, R. 1995, MNRAS, 272, 423

Colina, L., Gonzalez Delgado, R., Mas-Hesse, J. M., \& Leitherer, C. 2002, ApJ, 579, 545

Davies, R., Genzel, R., Tacconi, L., Mueller Sanchez, F., \& Sternberg, A. 2006a, preprint (astro-ph/0612009)

Davies, R. I., et al. 2006b, ApJ, 646, 754

Elitzur, M., \& Shlosman, I. 2006, ApJ, 648, L101

Fabian, A. C., Barcons, X., Almaini, O., \& Iwasawa, K. 1998, MNRAS, 297, L11

Fathi, K., Storchi-Bergmann, T., Riffel, R. A., Winge, C., Axon, D. J., Robinson, A., Capetti, A., \& Marconi, A. 2006, ApJ, 641, L25

Genzel, R., et al. 1998, ApJ, 498, 579

González Delgado, R. M., Heckman, T., Leitherer, C., Meurer, G., Krolik, J., Wilson, A. S., Kinney, A., \& Koratkar, A. 1998, ApJ, 505, 174

Gorjian, V., Werner, M. W., Jarrett, T. H., Cole, D. M., \& Ressler, M. E. 2004 , ApJ, 605, 156

Grossan, B., Gorjian, V., Werner, M., \& Ressler, M. 2001, ApJ, 563, 687

Hony, S., Van Kerckhoven, C., Peeters, E., Tielens, A. G. G. M., Hudgins, D. M., \& Allamandola, L. J. 2001, A\&A, 370, 1030

Houck, J. R., et al. 2004, ApJS, 154, 18

Imanishi, M. 2002, ApJ, 569, 44 2003, ApJ, 599, 918

Imanishi, M., \& Wada, K. 2004, ApJ, 617, 214

Jaffe, W., et al. 2004, Nature, 429, 47

Laurent, O., Mirabel, I. F., Charmandaris, V., Gallais, P., Madden, S. C., Sauvage, M., Vigroux, L., \& Cesarsky, C. 2000, A\&A, 359, 887

Le Floc'h, E., Mirabel, I. F., Laurent, O., Charmandaris, V., Gallais, P., Sauvage, M., Vigroux, L., \& Cesarsky, C. 2001, A\&A, 367, 487

Leitherer, C., et al. 1999, ApJS, 123, 3

Lord, S. D. 1992, NASA Technical Memor. 103957

Lutz, D., Spoon, H. W. W., Rigopoulou, D., Moorwood, A. F. M., \& Genzel, R. 1998, ApJ, 505, L103

Maoz, D., Nagar, N. M., Falcke, H., \& Wilson, A. S. 2005, ApJ, 625, 699

Mason, R. E., Geballe, T. R., Packham, C., Levenson, N. A., Elitzur, M., Fisher, R. S., \& Perlman, E. 2006, ApJ, 640, 612

Miller, J. S., \& Goodrich, R. W. 1990, ApJ, 355, 456

Moorwood, A. F. M. 1986, A\&A, 166, 4

Moran, E. C., Barth, A. J., Kay, L. E., \& Filippenko, A. V. 2000, ApJ, 540, L73

Mueller Sánchez, F., Davies, R. I., Eisenhauer, F., Tacconi, L. J., Genzel, R., \& Sternberg, A. 2006, A\&A, 454, 481
Nemmen, R. S., Storchi-Bergmann, T., Yuan, F., Eracleous, M., Terashima, Y., \& Wilson, A. S. 2006, ApJ, 643, 652

Nenkova, M., Ivezić, Ž., \& Elitzur, M. 2002, ApJ, 570, L9

Packham, C., Radomski, J. T., Roche, P. F., Aitken, D. K., Perlman, E., AlonsoHerrero, A., Colina, L., \& Telesco, C. M. 2005, ApJ, 618, L17

Peeters, E., Spoon, H. W. W., \& Tielens, A. G. G. M. 2004, ApJ, 613, 986

Perlman, E. S., Sparks, W. B., Radomski, J., Packham, C., Fisher, R. S., Piña, R., \& Biretta, J. A. 2001, ApJ, 561, L51

Phillips, M. M., Pagel, B. E. J., Edmunds, M. G., \& Diaz, A. 1984, MNRAS, 210, 701

Prieto, M. A., Maciejewski, W., \& Reunanen, J. 2005, AJ, 130, 1472

Prieto, M. A., et al. 2004, ApJ, 614, 135

Radomski, J. T., Piña, R. K., Packham, C., Telesco, C. M., De Buizer, J. M., Fisher, R. S., \& Robinson, A. 2003, ApJ, 587, 117

Rigopoulou, D., Spoon, H. W. W., Genzel, R., Lutz, D., Moorwood, A. F. M., \& Tran, Q. D. 1999, AJ, 118, 2625

Risaliti, G., et al. 2006, MNRAS, 365, 303

Roche, P. F., \& Aitken, D. K. 1985, MNRAS, 215, 425

Roche, P. F., Aitken, D. K., Smith, C. H., \& Ward, M. J. 1991, MNRAS, 248, 606

Roche, P. F., Packham, C., Aitken, D. K., \& Mason, R. E. 7, MNRAS, 375, 99

Roche, P. F., Packham, C., Telesco, C. M., Radomski, J. T., Alonso-Hererro, A., Aitken, D. K., Colina, L., \& Perlman, E. 2006, MNRAS, 367, 1689

Rodríguez-Ardila, A., \& Viegas, S. M. 2003, MNRAS, 340, L33

Sako, S., et al. 2003, PASP, 115, 1407

Siebenmorgen, R., Krügel, E., \& Spoon, H. W. W. 2004, A\&A, 414, 123

Snijders, L., van der Werf, P. P., Brandl, B. R., Mengel, S., Schaerer, D., \& Wang, Z. 2006, ApJ, 648, L25

Soifer, B. T., Bock, J. J., Marsh, K., Neugebauer, G., Matthews, K., Egami, E., \& Armus, L. 2003, AJ, 126, 143

Storchi-Bergmann, T., Baldwin, J. A., \& Wilson, A. S. 1993, ApJ, 410, L11

Storchi-Bergmann, T., Nemmen, R. S., Spinelli, P. F., Eracleous, M., Wilson, A. S., Filippenko, A. V., \& Livio, M. 2005, ApJ, 624, L13 (SB05)

Storchi-Bergmann, T., Raimann, D., Bica, E. L. D., \& Fraquelli, H. A. 2000, ApJ, 544, 747

Storchi-Bergmann, T., et al. 2003, ApJ, 598, 956

Tacconi-Garman, L. E., Sturm, E., Lehnert, M., Lutz, D., Davies, R. I., \& Moorwood, A. F. M. 2005, A\&A, 432, 91

Telesco, C. M., \& Gatley, I. 1981, ApJ, 247, L11

Telesco, C. M., Pina, R. K., Hanna, K. T., Julian, J. A., Hon, D. B., \& Kisko, T. M. 1998, Proc. SPIE, 3354, 534

Terashima, Y., Iyomoto, N., Ho, L. C., \& Ptak, A. F. 2002, ApJS, 139, 1

Terashima, Y., \& Wilson, A. S. 2003, ApJ, 583, 145

Tran, H. D. 2001, ApJ, 554, L19

van Diedenhoven, B., Peeters, E., Van Kerckhoven, C., Hony, S., Hudgins, D. M., Allamandola, L. J., \& Tielens, A. G. G. M. 2004, ApJ, 611, 928

Voit, G. M. 1992, MNRAS, 258, 841

Wada, K., \& Norman, C. A. 2002, ApJ, 566, L21

Watabe, Y., \& Umemura, M. 2005, ApJ, 618, 649

Whysong, D., \& Antonucci, R. 2004, ApJ, 602, 116

Young, S., Hough, J. H., Efstathiou, A., Wills, B. J., Bailey, J. A., Ward, M. J., \& Axon, D. J. 1996, MNRAS, 281, 1206

Zier, C., \& Biermann, P. L. 2002, A\&A, 396, 91 